Field report

\title{
Trends in Paraquat Poisoning in Japan - Viewed from Surveys on Clinical Cases
}

\author{
Hiroshi Nagami, Fumio Maejima, Yoshio Nishigaki and Shusuke Natsukawa \\ Division of Health Care, Saku Central Hospital, Japan
}

\begin{abstract}
Objective: Paraquat poisoning occurs worldwide, and both the fatality rate and the number of deaths are out of the ordinary. Japan is one of the few countries in the world that have replaced $24 \%$ products with $5 \%$ products. This report is an attempt to introduce information about paraquat poisoning in Japan.

Methods: The Japanese Association of Rural Medicine has carried out extensive surveys on clinical cases of pesticide poisoning at its 122 member installations since 2007. Of all the findings, we have picked out and evaluated those concerned with paraquat poisoning. Findings: Twenty-two out of 28 persons who attempted to commit suicide with paraquat died in 2007-2011. The rate of deaths from suicide attempts with paraquat stands at somewhere near $80 \%$ or so even with $5 \%$ products, so it appears that the attempts to reduce the death rate did not have the intended effect even with products having a lower concentration.

Conclusion: The "outcome prediction line" propounded in 1979 still stands today, while a wide variety of therapies have been studied. It seems that paraquat should at least be classified into Class Ib (highly hazardous) given that the mortality remains high and efficacious therapies remain elusive.
\end{abstract}

Key words: paraquat, suicide, mortality in Japan

(J Rural Med 2013; 8(2): 228-232)

\section{Introduction}

The many deaths arising from suicide attempts with pesticides are considered a grave social issue in the rural Asian setting $^{1-3)}$. Above all, the mortality rate and the number of deaths, which are particularly high for suicide attempts with paraquat $(\mathrm{Pq})$, pose serious problems $\mathrm{s}^{4-7)}$.

In the 1980 s, grave social issues were posed from poi-

Received: 24 May 2013, Accepted: 28 June 2013

Correspondence to: Hiroshi Nagami, Division of Health Care, Saku

Central Hospital, 197, Usuda, Saku, Nagano 384-0301, Japan

E-mail: nagami@dia.janis.or.jp

The authors state that they have no conflict of interest. soning with $\mathrm{Pq}$, as there arose not just deaths from suicide attempts but also homicide attempts and occupational exposure. In 1986, sale of $24 \%$ products was suspended, and they were replaced with $5 \%$ products. Even so, there has been no end to suicide attempts with $\mathrm{Pq}$, which poses issues for emergency care.

A special team for studies on pesticide poisoning was established in the Japanese Association of Rural Medicine (JARM) in 1992, and 102 medical facilities under its wings began a nationwide pesticide poisoning survey in 1996. Nagami et al. ${ }^{8}$ came out with an analytical report on the data gathered on Pq poisoning from 1998 to 2003. Taking another look at its research system, the JARM established the Special Research Project and Pesticide Poisoning Panel. This group reopened surveys on clinical cases in 2007. Here, the authors assessed the present state of Pq poisoning in Japan by analyzing the clinical cases amassed in 2007-2011.

\section{Methods}

The JARM's Special Research Project and Pesticide Poisoning Panel began to conduct surveys in 2007 at the 122 JARM-affiliated facilities across the nation. With the start of the fiscal year legally set as April 1 in Japan, reports on clinical cases at the first visits in the previous fiscal year were collected in mid-April.

The clinical-case questionnaire included, sex, age, date and time of exposure, date and time of the first visit, date of treatment end, vocation, main complaint, conditions of exposure, route of exposure, cause of poisoning, causative pesticide (product name, name of active ingredient and/or name of classification), intake of poison in amount, concentration of ingredient, diagnosis, degree of severity, treatment, pathological change and sequelae, among others.

As regards pathological changes due to suicide committed with Pq, their relations to sex and age were checked and compared with product-specific pathological changes ${ }^{8)}$ sur- 
Table 1 Outcomes of paraquat suicide cases in Japan - Survey of clinical cases by the Japanese Association of Rural Medicine in 2007-2011, by gender and age

\begin{tabular}{llcc}
\hline \multicolumn{2}{l}{ Characteristics } & Death & Survival \\
\hline Gender & Male & 6 & $2^{\mathrm{a}}$ \\
& Female & 16 & $3^{\mathrm{b}}$ \\
\hline Age & $20-39$ & 1 & $1^{\text {c }}$ \\
& $40-59$ & 8 & $1^{\mathrm{c}}$ \\
& $60-79$ & 10 & $4^{\mathrm{d \dagger}}$ \\
& $80-99$ & 3 & $0^{\mathrm{d}}$ \\
\hline
\end{tabular}

a vs. b: not significant ( $\mathrm{p} \geq 0.05$ ); Fisher's exact test.

${ }^{\dagger}$ For statistical analysis, ages are classified into two groups $-20-59$ and $60-99$ years old. c vs. d: not significant $(\mathrm{p} \geq 0.05)$; Fisher's exact test.

veyed in 1998-2003.

Along with the questionnaire on clinical cases, a "facility-specific questionnaire" was sent out to the JARM-affiliated facilities, which were asked to report the total numbers of outpatients and inpatients each year. The collected data were checked against the Hospital Report ${ }^{9)}$ put out by the Ministry of Health, Labour and Welfare, and the reported numbers of deaths were compared with those in the Ministry's Vital Statistics of Japan ${ }^{10)}$.

This study complied with the "Ethical Guideline for Epidemiological Study"11) notification of the Japanese Ministry of Health, Labour and Welfare, and obtained the approval of the Ethics Committee of the JARM. The statistical processing was done using Fisher's exact test with IBM SPSS ver. 19.

\section{Results}

\section{Sex-, age- and product-specific pathological changes}

Table 1 shows pathological changes by sex and age during the five years extending from 2007 to 2011 . The products were unexceptionably the liquids that contained $5 \% \mathrm{Pq}$ and $7 \%$ diquat $(\mathrm{Dq})(5 \% \mathrm{Pq}+7 \% \mathrm{Dq})$. There was no significant difference by pathological change (survival/death), sex and age.

In Table 2, product-specific pathological changes are compared in regard to cases of suicide with Pq products between 1998-2003 and 2007-2011. Eight cases of suicide attempts with $24 \%$ Pq were reported in 1998-2003, and all of them died. There were also reports on 15 cases of Pq poisoning in which the names of the products were unknown, and all of these cases also died. When it comes to $5 \% \mathrm{Pq}+7 \% \mathrm{Dq}$, the mortality rate stood at $81 \%$ and $79 \%$ in $1998-2003$ and 2007-2011, respectively. No significant difference was observed in the mortality rate among $24 \%$ products, products with unknown trade names and $5 \%$ products.
Table 2 Outcomes of paraquat suicide cases in Japan - Survey of clinical cases by the Japanese Association of Rural Medicine in 1998-2003 and 2007-2011, by product and year

\begin{tabular}{llcc}
\hline Year/product & Death & Survival \\
\hline $1998-2003$ & $24 \% \mathrm{Pq}$ & 8 & $0^{\mathrm{a}}$ \\
& Unknown & 15 & $0^{\mathrm{b}}$ \\
& $5 \% \mathrm{Pq}+7 \% \mathrm{Dq}$ & 39 & $9^{\mathrm{c}}$ \\
\hline $2007-2011$ & $5 \% \mathrm{Pq}+7 \% \mathrm{Dq}$ & 22 & $6^{\mathrm{d}}$ \\
\hline
\end{tabular}

Pq: paraquat, Dq: diquat. a vs. c, b vs. c, c vs. d: not significant ( $\mathrm{p} \geq 0.05)$; Fisher's exact test.

\section{Scale of this survey}

The total rate of inpatients at 94-97 JARM-affiliated facilities (with a recovery rate of $77-80 \%$ ) to that of inpatients across Japan, which was obtained from the statistics in the Ministry of Health, Labour and Welfare's Hospital Report, was $1.7-1.8 \%$. That of outpatients was $2.7-3.0 \%$. The rate of deaths tabulated in this survey to the number of deaths caused mainly by exposure to pesticides across Japan, as reported in the Vital Statistics of Japan, stood at 1.9-2.4\%.

Presumably, the rate of findings in this report as against the whole of Japan may come with the range of the aforementioned three indices, or somewhere between $1.7 \%$ and $3.0 \%$.

\section{Discussion}

\section{Sex- and age-specific pathological changes}

As regards the 28 cases poisoned with $\mathrm{Pq}$ and resulting in death, whose data were gathered from 2007 to 2011, no significant difference was observed in terms of sex, age and mortality, as indicated in Table 1.

Nagami et al. ${ }^{8)}$ report about 71 clinical cases in the period of 1998 to 2003 and found that there was no significant sex-specific difference but that the mortality rates were higher among people aged 60 and over than those among people aged 59 and under. When it comes to clinical cases in 2007-2011, no significant difference may be observed, as the number was small. Data on clinical cases should be collected for the sake of reassessment.

Paraquat products with a lower concentration and mortality rate

As shown in Table 2, no significant product-specific difference was observed between the period of six years from 1998 to 2003 and that of five years from 2007 to 2011.

A check of other surveys and research in Japan revealed that Chishiro ${ }^{12}$ reported that the use of products with a low concentration had begun, but no significant drop was ob- 


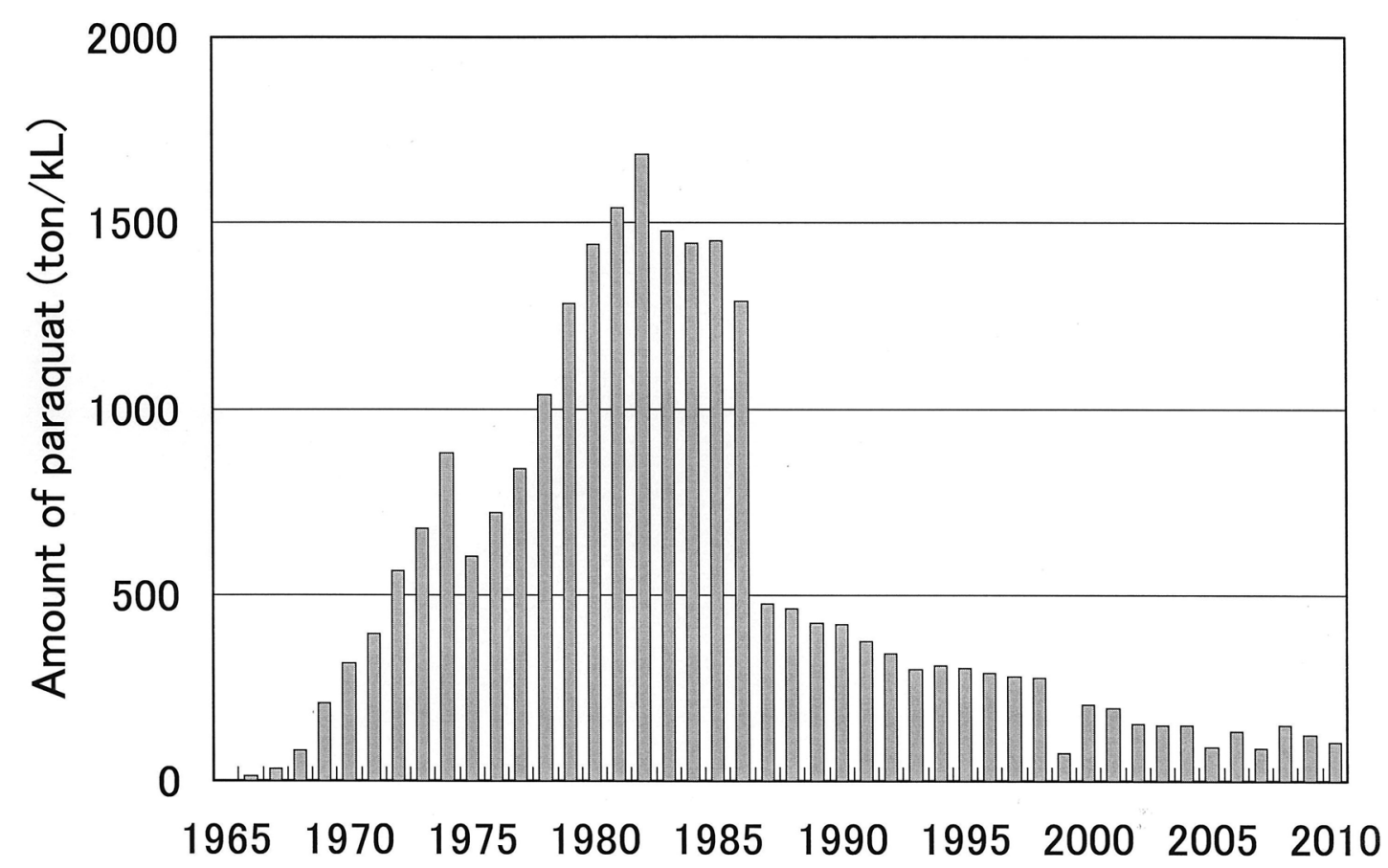

Figure 1 Trends of paraquat sales in Japan surveyed by the National Institute for Environmental Studies ${ }^{14}$.

served in the mortality rate. In an investigation of 47 clinical cases at Hiraka General Hospital, Ichinose et al. ${ }^{13)}$ did not observe any significant difference in the mortality rate between products with a high concentration and those with a low concentration.

The shift from $24 \%$ products to $5 \%$ products appears to have failed to effectively decreased the number of deaths due to suicide attempts with Pq.

\section{Scale of this survey and limitation}

By comparing the total number of inpatients, that of outpatients and the number of deaths in 2007-2010 in the JARM survey with the national statistics, the rate in this survey may be somewhere between $1.7 \%$ and $3.0 \%$. In the JARM survey in 1998-2003, a similar comparison was also performed. The percentage was estimated to be $1.5-2.8 \%$ for the three indices combined, which is virtually the same as in 2007-2010.

Presumably, data on the majority of cases admitted to hospitals were collected, so almost all cases of individuals committing suicide with Pq must have been reported.

Nonetheless, the degree to which minor cases treated at outpatient clinics were grasped is open to question. The authors intend to reexamine this issue with electronic clinical charts, the sophistication of which has been remarkable in recent years.

\section{Amount of sales and number of clinical cases}

The National Institute for Environmental Studies has prepared a database on the shipment of toxic chemicals. On the basis of that data ${ }^{14}$, the amount of Pq shipped domestically is shown in Figure 1. Shipments of Pq reached 1,500 tons a year in the 1980s, thus giving rise to not just suicide but also homicide and death by occupational exposure.

This development was considered a grave social issue in Japan. Due to pressure from the public, sale of $24 \% \mathrm{Pq}$ was suspended, and $24 \% \mathrm{Pq}$ was subsequently replaced with $5 \% \mathrm{Pq}+7 \% \mathrm{Dq}$.

As is discernible from cases in which $24 \% \mathrm{Pq}$ was used for suicide in 1998-2003, storage of pesticides in the barns of farmhouses for more than 10 years is not rare in Japan.

Not with this practice in mind, though, the sales of $\mathrm{Pq}$ were cut in half from 1998-2003 to 2007-2010, and the number of clinical cases dropped from 71 cases in 19982003 to 28 cases in 2007-2011, suggesting that there were correlations between the amount of sales and the number of clinical cases.

Number of deaths and amount of sales listed in national statistics

Citing data from the Vital Statistics in Japan prepared by the Ministry of Health, Labour and Welfare ${ }^{10)}$, Figure 2 numerically shows deaths caused by exposure to pesticides. Before 1978, deaths had remained classified into a single 


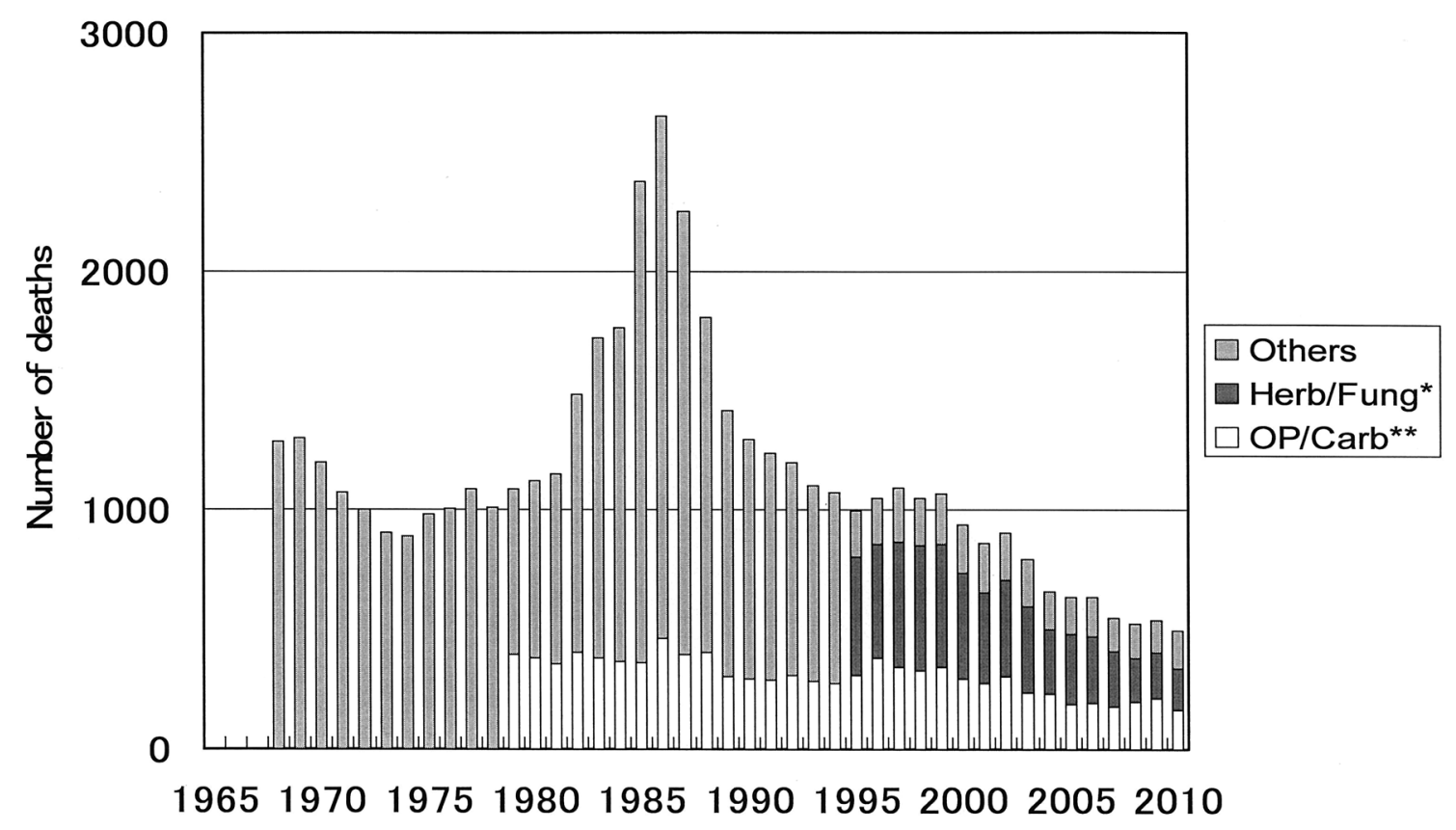

Figure 2 Trends in number of deaths by pesticide exposure according to Vital Statistics of Japan ${ }^{10)}$.

* Herb/Fung: herbicide and fungicide. ** OP/Carb: organophosphorus and carbamate.

category, but from 1979 to 1994 , they were classified into two categories - those caused by exposure to organophosphorus and carbamate insecticides and those caused by other pesticides. Naito ${ }^{15)}$ inferred that $\mathrm{Pq}$ was responsible for most of the deaths described as being caused by "other pesticides." In 1996 and subsequent years, the pesticides were further subdivided, and Figure 2 shows only those caused by herbicides and fungicides.

The figures shown here decreased by almost half from 1998-2003 to 2007-2011 and it is suggested that these figures, as in the preceding paragraph, are correlated with the amount of Pq sold.

\section{Outcome prediction line}

As regards the 21 cases for which the $\mathrm{Pq}$ concentration of plasma was measured and reported, Nagami et al..$^{8}$ illustrated correlations among time from swallowing to bloodsampling, Pq concentration and outcome and ascertained that cases of death were distributed above the outcome prediction line propounded by Alex T. Proudfoot ${ }^{16)}$ in 1979 and that cases of survival were under the line.

Chishiro ${ }^{12)}$ and Ichinose et al. ${ }^{13)}$ argued that the Proudfoot line is useful. Senarayhna et al. ${ }^{17}$ in Sri Lanka studied 451 clinical cases in 2002-2006 and reported that the Proudfoot line and several other outcome prediction lines proposed later proved effective.

A great number of studies on therapies for Pq have ap- peared in the last several decades, but it has been argued that they have not proved to be effective in decreasing the mortality rate ${ }^{4}$.

\section{Classification of acute toxicity}

The WHO International Programme on Chemical Safety ${ }^{18)}$ (WHO-IPCS) has classified pesticides according to degrees of acuteness. This classification has served to gradually reduce registrations of highly toxic pesticides, thus contributing to a drop in the outbreak of pesticide poisoning.

Weighing $150 \mathrm{mg} / \mathrm{kg}, \mathrm{LD}_{50}$ in Pq-poisoned rats is categorized in Class II (moderately hazardous) under WHOIPCS $^{18)}$. According to the Extension Toxicology Network ${ }^{19)}$ managed by Cornell University and three other American universities, on the other hand, the values of $\mathrm{LD}_{50}$ are smaller for mammals other than rats, such as in monkeys, cats and cows, the $\mathrm{LD}_{50}$ values of which are $50 \mathrm{mg} / \mathrm{kg}, 48 \mathrm{mg} / \mathrm{kg}$ and $50-75 \mathrm{mg} / \mathrm{kg}$, respectively.

When it comes to humans, Mergel $\mathrm{M}^{20)}$ set the $\mathrm{LD}_{50}$ at 35 $\mathrm{mg} / \mathrm{kg}$, whereas 4 farmers Pty. Ltd. ${ }^{21)}$ set it at $30 \mathrm{mg} / \mathrm{kg}$. As effective therapies have yet to worked out, Pq should at least be put into Class Ib (highly hazardous).

\section{Conclusion}

Japan is one of the few countries that has experienced in distributing and using $5 \%$ products of $\mathrm{Pq}$, a herbicide. The 
authors studied Pq poisoning cases of attempted suicide on the basis of surveys performed on clinical cases of pesticide poisoning by JARM-affiliated hospitals.

The mortality rate for suicide attempts with Pq products is somewhere around $80 \%$ even for $5 \%$ products, so the attempts to decrease the mortality rate with Pq products having lower concentration did not prove to be effective.

It has also been suggested that trends in the number of deaths from suicidal attempts with $\mathrm{Pq}$ and the amount of $\mathrm{Pq}$ shipped are correlated.

For clinical cases in which the Pq concentrations in the plasma were measured, the outcome prediction line proposed by Alex T. Proudfoot in 1979 was useful till 2000 or so.

A wide variety of therapies have been studied in the last several decades, to be sure, but few therapies have been developed to decrease the mortality rate in an effective manner. As efficaciuos therapies for $\mathrm{Pq}$ poisoning remain elusive, $\mathrm{Pq}$ should at least be categorized into Class Ib (highly hazardous).

\section{Acknowledgment}

We would like to thank the physicians and other medicals staff working at the JARM-affiliated medical institutions for their cooperation. This study was conducted as one of the JARM's Special Research Projects and as part of its pesticide poisoning group's activity. We declare that we have no conflict of interest.

\section{References}

1. Zhang J, Li Z. Suicide means used by Chinese rural youths: A comparison between those with and without mental disorders. J Nerv Ment Dis. 2011; 199: 410-415. [Medline]

2. Wu KC, Chen YY, Yip PSF. Suicide methods in Asia: Implications in suicide prevention. Int. J. Environ. Res. Public Health. 2012; 9: 1135-1158. [Medline]

3. Pearson M, Konradsen F, Gunnell D, et al. A communitybased cluster randomized trial of safe storage to reduce pesticide self-poisoning in rural Sri Lanka: study protocol. BMC Public Health 2011; 11: 879. [Medline]

4. Gawarammana IB, Buckley NA. Medical management of paraquat ingestion. Br J Clin Pharmacol. 2011; 72: 745-757. [Medline]

5. Neumeister L, Isenring R. Paraquat Unacceptable health risks for users. 3rd Edition. Berne Declaration, Zurich, 2011. Available from: http://www.evb.ch/cm_data/Paraquat_Unaccept ableHealthRisk_3rdEdition_2011_mb_print.pdf. (accessed: 2013/05/24).

6. Wesseling C, Corriols M, Bravo V. Acute pesticide poisoning and pesticide registration in Central America. Toxicol Appl
Pharmacol 2005; 207: 697-705. [Medline]

7. Lee WJ, Cha ES. Overview of pesticide poisoning in South Korea. J Rural Med 2009; 4: 53-58.

8. Nagami H, Nishigaki Y, Matsushima S, et al. Paraquat poisoning in Japan: a hospital-based survey. J Rural Med 2007; 2: 85-92.

9. Ministry of Health, Labour and Welfare. Hospital Report. Ministry of Health, Labour and Welfare, Tokyo, 2012. (in Japanese). Available from: http://www.mhlw.go.jp/toukei/ list/80-1.html. (accessed: 2013/05/24).

10. Ministry of Health, Labour and Welfare. Vital statistics of Japan. Ministry of Health, Labour and Welfare, Tokyo, 2012. (in Japanese).

11. Ministry of Health, Labour and Welfare. Ethical guideline of epidemiological study. Ministry of Health, Labour and Welfare, Tokyo, 2008. (in Japanese). Available from: http://www.mhlw.go.jp/general/seido/kousei/i-kenkyu/ ekigaku/0504sisin.html. (accessed: 2013/05/24).

12. Chishiro T. Clinical aspect of accidental poisoning with paraquat. Asian Med J. 2000; 43: 114-120.

13. Ichinose A, Kimura K, Tabata M, et al. A Study over 21 years of 47 patient with paraquat intoxication in Hiraka General Hospital. Respiration Research. 2004; 23: 318-323 (in Japanese, Abstract in English).

14. National Institute for Environmental Studies. Paraquat. National Institute for Environmental Studies, Tsukuba, 2012. (in Japanese). Available from: http://db-out.nies.go.jp/kis-plus/ noyaku_php/noyaku_select.php?cas_id=1910-42-5. (accessed: 2013/05/24).

15. Naito H. Poisoning of Industrial Products, Gases, Pesticide, Drugs, and Natural Toxins. 2nd ed. Nankodo, Tokyo, 2001; 293-305 (in Japanese).

16. Proudfoot AT, Stewart MS, Levitt T, et al. Paraquat poisoning: significance of plasma-paraquat concentrations. Lancet. 1979; 2: 330-332. [Medline]

17. Senarathna L, Eddleston M, Wilks MF, et al. Prediction of outcome after paraquat poisoning by measurement of the plasma paraquat concentration. QJM 2009; 102: 251-259. [Medline]

18. International Programme on Chemical Safety. The WHO Recommended Classification of Pesticides by Hazard. International Programme on Chemical Safety, Geneva, 2010. Available from: http://www.who.int/ipcs/publications/pesticides hazard 2009.pdf. (accessed: 2013/05/24).

19. Extension Toxicology Network. Paraquat. Extension Toxicology Network, Ithaca, 1993. Available from: http://pmep.cce. cornell.edu/profiles/extoxnet/metiram-propoxur/paraquatext.html. (accessed: 2013/05/24).

20. Mergel M. Paraquat. 2010. Available from: http://toxipedia. org/display/toxipedia/Paraquat. (accessed: 2013/05/24).

21. 4farmers Pty. Ltd. Material Safety Data Sheet PARAQUAT 250 HERBICIDE. 4farmers Pty. Ltd., Welshpool, 2004 Available from: http://www.herbiguide.com.au/MSDS/ MPAR20_58734-1204.PDF. (accessed: 2013/05/24). 